\title{
Vietnam, ein Markt für deutsche Unternehmen?
}

\author{
Prof. Dr. Peter Gadow
}

\section{Einführung}

Dieser Artikel soll einen kurzen Überblick über die Marktchancen deutscher Unternehmen, insbesondere kleiner und mittlerer Unternehmen (KMU) in Vietnam geben. Aufgrund der Globalisierung infolge der Liberalisierung der Weltwirtschaft sowie neuer technischer Möglichkeiten von Kommunikation und Logistik müssen gerade diese Unternehmen verstärkt ihre Märkte in der gesamten Welt suchen und finden. Besonders die neuen Bundesländern haben es in den klassischen gesättigten Märkten schwer, einen Marktanteil zu gewinnen.

Für ein Unternehmen gibt es prinzipiell zwei Hauptmöglichkeiten einen Marktanteil zu erreichen:

1. durch Innovationen, d. h. durch neue Produkte und Leistungen,

2. durch Erschließung neuer Marktregionen.

Im weiteren sei nur der zweite Weg mit Einschränkung auf Südostasien und dem Schwerpunkt Vietnam betrachtet.

Warum ist Vietnam für deutsche Unternehmen interessant? In Deutschland leben ca. 60.000 bis $100.000 \mathrm{Vi-}$ etnamesen, in Vietnam vielleicht 100.000 Vietnamesen mit Deutschkenntnissen und einige Tausende Hochschulabsolventen, die in Deutschland studierten. Damit existiert in Vietnam eine hohe Akzeptanz für Deutschland. Das bedeutet auch eine hohe Akzeptanz für deutsche Produkte und eine Zusammenarbeit mit deutschen Unternehmen. Die meisten jetzt in Vietnam lebenden Vietnamesen mit Deutschkenntnissen haben in der ehemaligen DDR studiert oder als Gastarbeiter gearbeitet. Somit sind die Kontakte und Kenntnisse häufig sehr stark auf die „Neue Bundesländer“ (NBL) bezogen. Viele dieser Vietnamesen kamen aus Nord- und Mittelvietnam. Heute kann man durchaus in einigen Ministerien, Großunternehmen und leitenden Positionen Vietnamesen antreffen, die in Deutschland studierten und mit denen man Verträge in deutsch verhandeln kann. Zusätzlich spricht für Vietnam seine zentrale geographische Lage in Südostasien. Auf der Karte Süidostasiens (Abb. 1) sieht man sofort diese zentrale Lage, die in der Zukunft wirtschaftlich und politisch von Deutschland genutzt werden sollte. Ein Vorreiter könnte die deutsche Wirtschaft in enger Verbindung mit der deutschen Wissenschaft sein. Hier läßt besonders durch KMU ein Einstieg finden, sowie eine Basis für ganz Südostasien aufbauen. Für Unternehmen aus den NBL - im wesentlichen $\mathrm{KMU}$ - mit ihren Erfahrungen des Übergangsprosses zur Marktwirtschaft kommt hinzu, daß sie und ihre Mitarbeiter die Schwierigkeiten vietnamesischer Unterneh- men und Institutionengut verstehen, durchläuft Vietnam zur Zeit ebenfalls einen Transformationsprozeß zu einem marktwirtschaftlichen orientierten System.

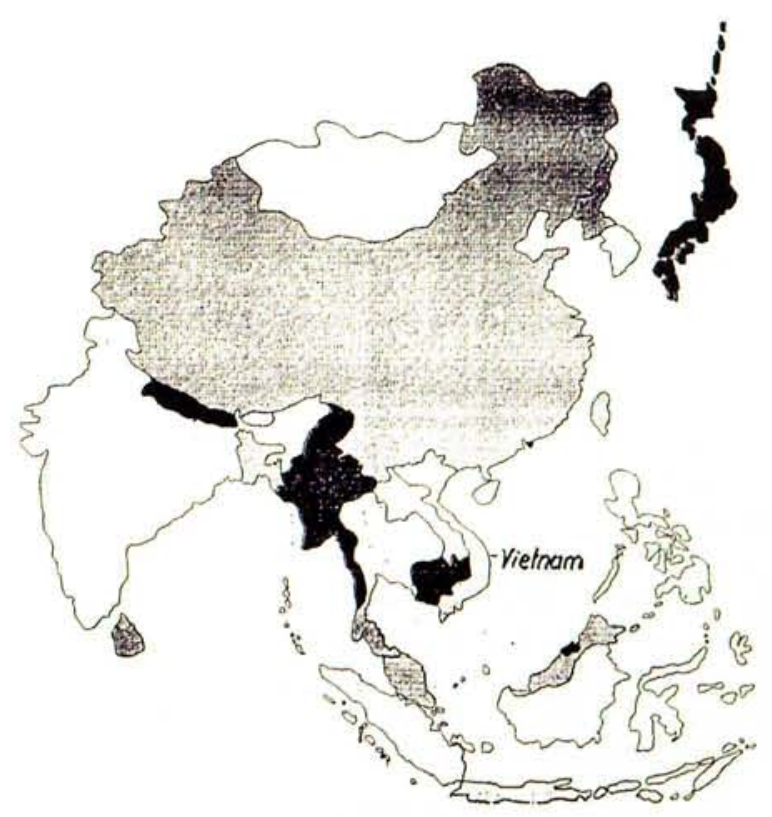

Abb. 1: Südostasien

\section{Vietnam - Allgemein}

Die tabellarische Zusammenfassung der wichtigsten Strukturdaten (Tab. 1) sowie die Vietnam-Karte (Abb. 2) geben einen kurzen Überblick.

\begin{tabular}{|l|l|}
\hline Fläche & $329500 \mathrm{~km}^{2}$ \\
\hline Bevölkerung & $74 \mathrm{Mio}$. \\
\hline Bevölkerungswachstum & $2,4 \%$ \\
\hline Lebenserwartung & 67 Jahre \\
\hline Stadtbevölkerung & $22 \%$ \\
\hline Alphabetisierung & $88 \%$ \\
\hline Bruttoinlandsprodukt [BIP] & 20,9 Mill. \$ \\
\hline Wachstumsrate & $9,3 \%$ \\
\hline BIP-Pro-Kopf & 272 \$ \\
\hline Inflationsrate & $4,5 \%$ \\
\hline Arbeitslosigkeit & $20 \%$ \\
\hline
\end{tabular}

Tab.1: Strukturdaten Vietnam Jahr 1996 12 


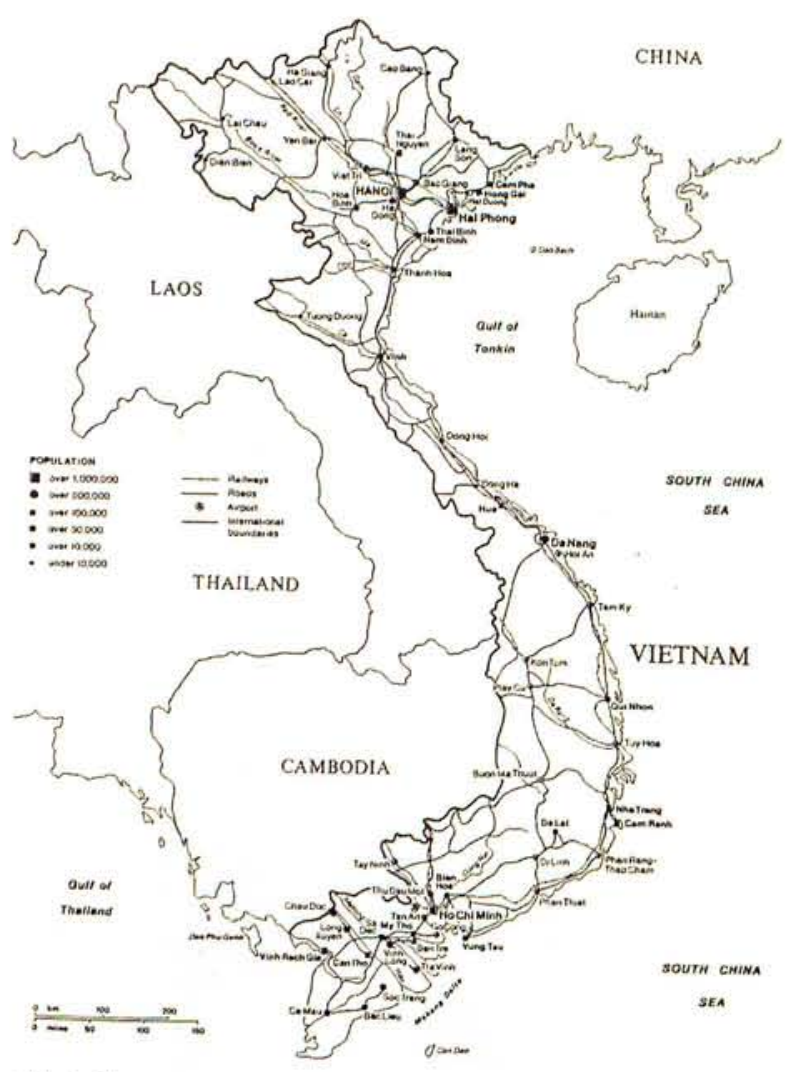

Abb. 2: Vietnam

- Vietnam als sehr langgestreckter Kïstenstaat mit einer ca. $3.500 \mathrm{~km}$ langen Küste liegt in Südostasien am Südchinesischen Meer. Es hat Staatsgrenzen im Norden mit China, in der Mitte mit Laos sowie im Süden mit Kambodscha. Geographisch gliedert sich Vietnam in drei große Zonen.

- Der Norden mit den Stromgebieten des Roten (Song Hong) und des Schwarzen Flusses (Song Da) stellt das Kernland Vietnams dar. Dieses Gebiet ist eines der dichtesten besiedelten Gebiete der Erde (mit über 1.000 Einwohner pro $\mathrm{km}^{2}$ ). Das Klima ist subtropisch.

- Mittelvietnam ist im wesentlichen ein Bergland, teilweise ein nur sehr schmaler Küstenstreifen (wenige km breit). Ferner sind einige Hochplateaus eingelagert, die u. a. für Tee- und Kaffeeanbau genutzt werden.

- Der Süden ist ebenfalls eine Stromaufschwemmungsebene: Das etwa $70.000 \mathrm{~km}^{2}$ große Mekongdelta ist ein Labyrinth von Wasserarmen, zwischen denen sich Sümpfe, Sumpfwälder und Reisfelder ausdehnen. Es handelt sich um eines der besten Reisanbaugebiete der Erde.

Vietnam ist reich an Bodenschätzen. Ihre Lagerstätten befinden sich hauptsächlich im Norden und teilweise in Mittelvietnam. Damit lassen sich weiterhin drei große Wirtschaftsgebiete und Wirtschaftswachstumsdreiecke definieren (Abb. 3):

1. Im Norden: Hanoi - Quang Ninh - Hai Phong

2. In Mittelvietnam: Da Nang - Hue - Quang Ngai

3. Im Süden: Ho Chi Minh City $(\mathrm{HCMC})-$ Bien Hoa - Vung Tau

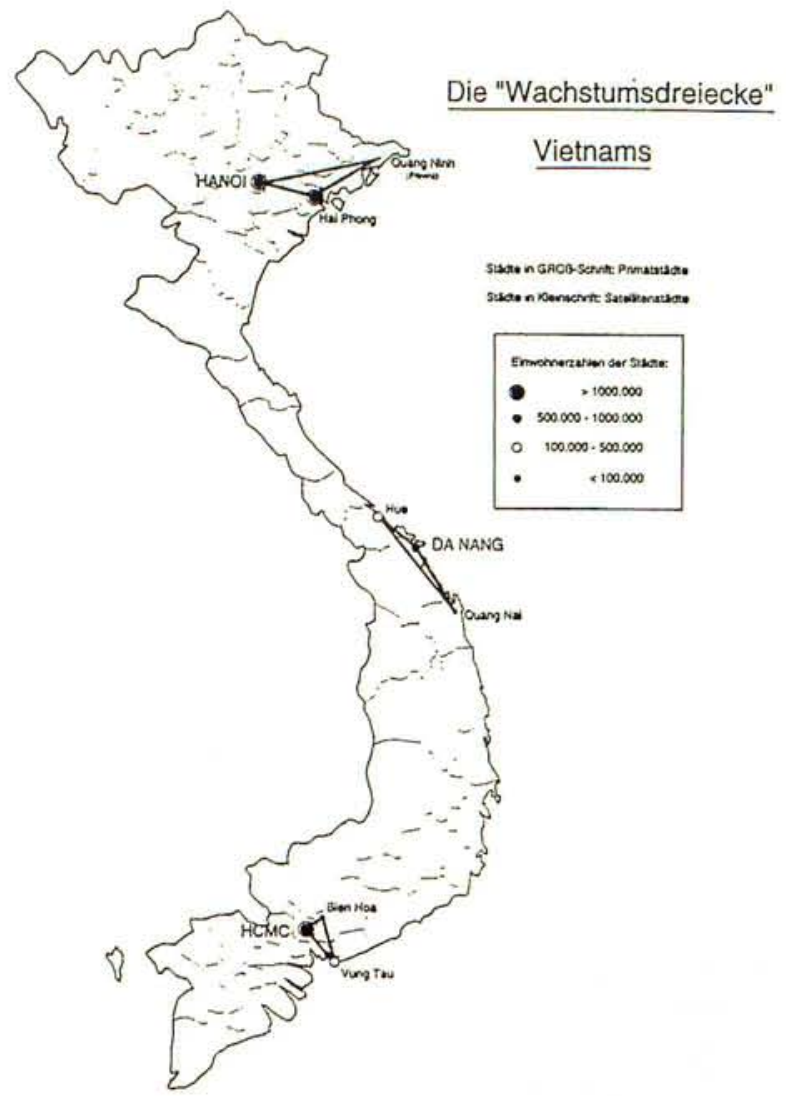

Abb. 3: Wachstumsdreiecke in Vietnam

\section{Wirtschaft}

Eine marktwirtschaftliche Entwicklung kann man etwa seit Einführung der sogenannten „Doi Moi“-Politik 1986 feststellen. Zuerst war sie in der Landwirtschaft wirksam. Sie führte dazu, daß Vietnam, von der damaligen Unterversorgung mit Reis ausgehend, heute der drittgrößte Reisexporteur der Welt ist. Damit ist in Vietnam die Grundversorgung der Bevölkerung gesichert. Der Staat hat sich aus der Nahrungsmittelerzeugung weitgehend zurückgezogen, dieser Sektor ist fast vollständig marktwirtschaftlich organisiert. Das besagt aber nicht, daß Handel und Nahrungsmittelindustrie voll marktwirschaftlich wirken. Hier spielt der Staat noch eine starke Rolle, d. h. er tritt in diesen Bereichen teilweise als planwirtschaftlich agierender Unternehmer auf. Die „Doi Moi“-Politik ist auch in der gewerblichen Wirtschaft wirksam. Eine Aufteilung der einzelnen Wirtschaftssektoren in bezug auf das BIP für das Jahr 1994 zeigt Abb. $4 .^{3}$

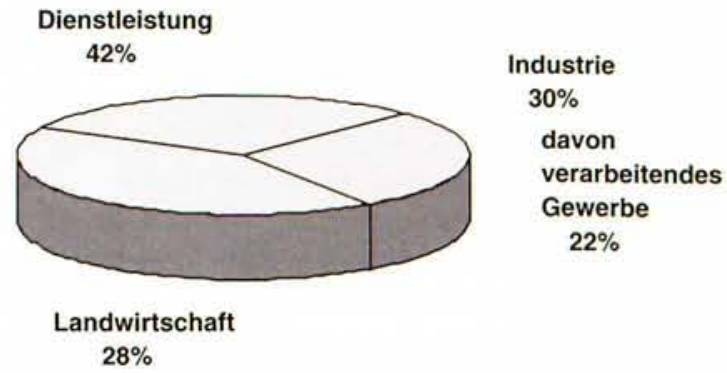

Abb. 4: Beitrag der Sektoren zum BIP in $\%$ 
Die Rolle des privaten Sektors in der Wirtschaft nimmt laufend zu. Für 1995 wurde offiziell angegeben, daß bei Dienstleistungen und Handel (einschließlich Gaststätten) der Anteil des privaten Sektors ca. $67 \%$ sei. Es geht dabei vorwiegend um Klein- und Kleinstbetriebe. Die 350.000 privaten Betriebe, die in den letzten Jahren gegründet wurden, beschäftigen schätzungsweise jeweils weniger als 30 Mitarbeiter. Die Zahl der privaten Unternehmen mit einem Investitionskapital von mehr als 20.000 US-Dollar wurde auf weniger als 1.200 geschätzt. ${ }^{4}$

Einen weitereren wichtigen Wirtschaftsfaktor stellt die zu erwartende Wachstumsrate des BIP dar. In der folgende Tabelle sind die Daten anderer wichtigen Länder mitaufgeführt, wobei die Raten für Indonesien, Malaysia, Philippinen und Thailand durch die suidostasiatische Finanzkrise noch nach unten korrigiert werden müssen.

\begin{tabular}{|l|r|r|r|r|}
\hline \multicolumn{6}{|c|}{$\begin{array}{c}\text { ASEAN BIP Wachstumsraten } \\
\text { (jährliche Wachstumsrate in \%) }\end{array}$} \\
\hline & 1995 & 1996 & 1997 & 1998 \\
\hline Indonesien & 8,2 & 7,8 & 8,0 & 7,9 \\
\hline Malaysia & 10,1 & 8,8 & 8,5 & 8,5 \\
\hline Philippinen & 4,8 & 5,5 & 6,0 & 6,5 \\
\hline Singapur & 8,8 & 7,0 & 7,5 & 8,0 \\
\hline Thailand & 8,7 & 6,7 & 6,1 & 6,6 \\
\hline Vietnam & 9,5 & 9,5 & 9,5 & 9,3 \\
\hline
\end{tabular}

Tab. 2: Jährliche Wachstumsraten des BIP für ausgewählte ASEAN Staaten ${ }^{5}$

Die Wachstumsraten im industriellen bzw. gewerblichen Bereich liegen wesentlich höher. Die jährliche landwirtschaftliche Wachstumsrate wird mit $4,5 \%$, die des Dienstleistungssektors wird mit einer $9 \%$-Rate geschätzt. Bei diesen Daten würde sich bei einer jährlichen Gesamtrate von 9,5\% für den industriellen Bereich eine Wachstumssteigerung von ca. $15 \%$ ergeben. ${ }^{6}$ In der Tabelle 3 werden weitere für die Beurteilung wichtigen Kennziffern zusammengefaßt.7

\begin{tabular}{|l|r|r|r|r|}
\hline & 1994 & 1995 & 1996 & $1997^{\circ}$ \\
\hline Bruttoauslandsverschuldung & 25 & 25,5 & & \\
\hline Schuldensdienstquote & $11,4 \%$ & $12,6 \%$ & & \\
\hline Exporte - Gesamt & 3,8 & 4,7 & $6,4^{8}$ & $8,3^{8}$ \\
\hline Importe - Gesamt & 4,4 & 4,7 & $10,1^{8}$ & $11,1^{8}$ \\
\hline Leistungsbilanz & $-0,2$ & $-0,1$ & $-3,1^{8}$ & $-2,1^{8}$ \\
\hline Exporte nach Deutschland & & $0,816^{7}$ & $0,929^{8}$ & $0,563^{\circ *}$ \\
\hline Importe aus Deutschland & & $0,340^{7}$ & $0,514^{8}$ & $0,218^{\circ}$ \\
\hline
\end{tabular}

Tab. 3: Zahlenspiegel der Wirtschaft (wenn nicht anders angegeben in Mrd. US-\$) * Prognose; “" Januar-Februar; 7) Märkte Ostasiens, 1995, Deutscher Sparkassenverbund S. 245; 8) Länderbericht-Vietnam, Okt. 1997, Berliner Bankgesellschaft

Vietnam, das fruiher wegen der besonderen politischen Beziehungen zum ehemaligen Ostblock im wesentlichen Kompensationsgeschäfte mit diesen Ländern durchführte, wickelt heute etwa $90 \%$ seines Außenhandels mit asiatischen und westlichen Ländern auf der Basis kon- vertibler Währungen ab. Die Exporte und Importe sind wertmäßig relativ gering. Aber selbst bei diesen geringen Summen liegt Deutschland nur an ca. 23. Stelle. In Tabelle 4 wird eine Übersicht über die getätigten Investitionen des Jahres 1996 gegeben. ${ }^{9}$

\begin{tabular}{|l|c|}
\hline Land & Investitionen [Mio US-S] \\
\hline Taiwan & 350 \\
\hline Hong Kong & 280 \\
\hline Singapur & 220 \\
\hline Südkorea & 200 \\
\hline Indonesien & 190 \\
\hline USA & 55 \\
\hline Frankreich & 45 \\
\hline England & 36 \\
\hline Deutschland & 18 \\
\hline
\end{tabular}

Tab. 4: Investitionen von ausgewählten Ländern im Jahre 1996

Von der Situation 1997 ausgehend ergeben sich folgende Wachstumsmärkte, die von der vietnamesischen Regierung besonders gefördert werden und im Plan 2000 verankert sind:

- Telekomsektor

- Stahlindustrie

- Verkehrssysteme

Für den Konsumguiterbereich läßt sich ein hoher Nachholbedarf an modischer Bekleidung ausmachen. Hier läge ein wichtiger Kooperationsbereich mit der deutschen Textil- und Bekleidungsindustrie. Ein hoher Nachholbedarf zeigt sich auch bei langlebigen Konsumgütern und bei Motorrädern, dem typischen individuellen Fortbewegungsmittel in Vietnam. So kann man in Hanoi von einem Nahverkehr kaum sprechen. Er wird nahezu ausschließlich vom Individualverkehr mit Fahrrädern und Kleinmotorrädern getragen.

Typisch für den Handel ist Kleinhandel in Form von Basarhandel. Es gibt keine Großhandelsstrukturen wie in den entwickelten Industrieländern, sondern Strukturen der staatsplanwirtschaftlich gelenkten Industrie im Sinne zentraler Beschaffungs- und Verteilungsstrukturen. Handelsbeziehungen müssen darauf aufbauen, dies ist aber nur mit vietnamesischen Partnern möglich, die in diesen Bereichen arbeiten oder sie nutzen.

Für Verbrauchsgüter ist der Schmuggelanteil sehr hoch. Er wird auf ca. 500 Mio. US-Dollar pro Jahr geschätzt. ${ }^{10}$ Vietnamesen haben als Kunden, allgemein gesprochen, eine ausgesprochene konservative Produkteinstellung. Dies wird z. B. bei der Unterhaltungselektonik oder auch beim Verkehrsmittel Motorrad deutlich. Der "Mercedes“ unter den Motorrädern ist das Modell „Honda“. Obwohl technisch und preislich nicht das günstigste Modell auf dem vietnamesischen Markt, wird es bevorzugt. Speziell für die in Vietnam bekannten Firmenmarken aus der ehemaligen DDR bietet sich hier eine Marktchance. Die im Ausland lebenden oder dort ausgebildeten Vietnamesen haben mit ihren Konsumerfahrungen und ihren Präferenzen von Produkten und Marken einen starken Einfluß auf Verwandte und Bekannte in Vietnam. Bei den 
starken Gruppenbeziehungen in Vietnam sowie in Südostasien überhaupt ist das ein Faktor, der zwingend zu beriicksichtigen ist.

In der folgenden Übersicht sind in Bezug auf Investitionsgüter die relevanten Steigerungsraten für das Jahr 1996 noch einmal zusammengestellt:

$\begin{array}{lr}\text { - Investitionen } & 25,0 \% \\ \text { - Importe } & 24,0 \% \\ \text { - BIP } & 9,3 \% \\ \text { - Landwirtschaft } & 4,5 \%\end{array}$

Unter Berüicksichtigung der oben genannten Schwerpunkte haben folgende in den nächsten Jahren Bereiche einen hohen Bedarf an:

- Anlagen

- Baustoffherstellung

- Baumaschinen

- Werkzeugmaschinen

- Fahrzeuge

- Telekommunikation

- Chemie (Petrochemie)

\section{Administrative Rahmenbedingungen}

Aus deutscher Sicht ergeben sich für die Geschäftstätigkeit in Vietnam einige wichtige Fragen, die schon am Anfang behandelt werden müssen:

- Doppelsteuerabkommen (Seit 1997 ist der Vertrag über das Doppelsteuerabkommen mit Vietnam wirksam.)

- Gewinnsteuer. Hier die wichtigsten Überblickdaten (Es sei darauf hingewiesen, daß speziell in der Anfangsphase von Firmengründungen in Vietnam noch günstigere Bedingungen vorliegen können):

- 2-15\% für Infrastruktur-, Rohstoff-Unternehmen, Schwerindustrie, Baustoff-, Chemie- und Maschinenbauindustrie;

- 3-19\% für wichtige Projekte, Tourismus;

- $20 \%$ bei Unternehmen mit mehr als 500 Beschäftigten, Kapitaleinlagen sind höher als 10 Mio US-\$ und 80\% Exportvolumen;

- 25\% für alle anderen Fälle.

- Zoll. Die Einfuhrzölle sind sehr komplex und an komplizierte Verfahren gebunden. Eine jährlich erneuerte Liste mit Zolltarifen ist die Grundlage der Zolleinstufung. Die Tarife sind sehr unterschiedlich. Einige Beispiele sollen dies verdeutlichen: Für Pkw ist der Zoll bei $160 \%$ und für Textilien $50 \%$. Geringere Zölle werden für Investitionsgüter erhoben, es kann hier auch eine teilweise Befreiung vom Zoll erfolgen. Gebrauchte Investitionsguiter werden abhängig vom Alter im allgemeinen hoch versteuert. Somit sind subjektive Einschätzungen des Zollgutes gegeben und machen das Verfahren sehr bürokratisch.

\section{Löhne und Ausbildung}

Grundsätzlich gibt es in Vietnam ein starkes Süd-Nordgefälle. Löhne und Kaufkraft sind im Süden mehr als $30 \%$ höher. Im weiteren werden nur die Verhältnisse in
Nord- und Mittelvietnam betrachtet. Aber grundsätzlich gilt das Folgende auch für Südvietnam. Realistisch ist von folgenden Lohnverhältnissen auszugehen: Facharbeiter in der Produktion verdienen 48 bis 60 US-Dollar pro Monat, Ingenieure 150 bis 500 US-Dollar. Die Nebenkosten betragen ca. 5 bis $10 \%$. Die etwas hoch erscheinenden Personalkosten sind auch unter dem Aspekt der Loyalität den Unternehmen gegenüber zu betrachten. Die Fluktuation der Mitarbeiter ist bei geringer Bezahlung besonders hoch. Spezielle Kenntnisse und Informationen werden schnell auf dem Arbeitsmarkt verwertet. Arbeitsrechtliche Vertragsabsicherungen haben meist wenig Wert, da gesetzliche Bedingungen für Arbeitsverträge oft nicht umsetzbar bzw. nicht vorhanden sind. Wird die mögliche Spanne der Personalkosten nach unten ausgenutzt, so treten Fluktuationen und Konflikte auf. Dies läßt sich anhand von Schwierigkeiten südkoreanischer und anderer südostasiatischer meist (Joint-Venture-)Unternehmen belegen, wo es zu Arbeitskämpfen gekommen ist. ${ }^{11}$

In Vietnam gibt es eine große Anzahl von Hochschulabsolventen, aber eine moderne Managementausbildung steht erst am Anfang des Aufbaues, so daß man in vielen Bereichen auf theoretisch gut ausgebildete Ingenieure zurüickgreifen muß, die intern zu schulen sind. Dies gilt besonders für moderne Organisationskenntnisse. Für deutsche Unternehmen ist die hohe Anzahl von deutschkundigen Vietnamesen interessant, darunter, besonders in Nordvietnam, viele mit einer Hochschulausbildung. Hervorhebenswert ist die hohe Lernbereitschaft der Vietnamesen.

\section{Rechtliche Rahmenbedingungen}

Grundsätzlich sind bei ausländischenUnternehmen folgende Unternehmensformen berïcksichtigt: ${ }^{12}$

- Repräsentationen

- Kooperationen mit vietnamesischen Partnern

- Joint-Venture-Unternehmen

- Tochtergesellschaften (100\%)

Die entsprechenden Unternehmensformen seitens der vietnamesischen Seite können sein:

- Staatliche Unternehmen. Sie sind im industriellen Bereich vorherrschend. Der Großhandel wird ebenfalls weitgehend über staatliche Unternehmen, wie z. B. Tochterunternehmen von Ministerien abgewikkelt. Hier ist die Bedeutung von Tochterunternehmen der Provinzregierungen zu beachten. Diese Unternehmen sind häufig rein privatwirtschaftlich organisiert und werden auch privatwirtschaftlich geführt. Ihr Vorteil liegt in ihrem Informationsvorsprung. Ein Nachteil ist die personelle Verflechtung mit den jeweiligen Staatsorganen.

- Halbstaatliche Unternehmen. Sie gehen nach dem gleichen Muster wie die staatliche Unternehmen vor, es gelten analoge Einschätzungen.

- Private Unternehmen. Dies sind zumindest in Nordund Mittelvietnam meist kleine Unternehmen mit einer geringen Anzahl von Mitarbeitern (ca. 30 und weniger). Diese KMU sind auch im Rahmen ihrer fi- 
nanziellen Möglichkeiten hoch flexibel. Beispielsweise sind bei diesen kleinen Unternehmen Finanzierungen in der Größenordnung von ca. 200.000 US-Dollar auch ohne Bankkredite möglich.

Weitere Möglichkeiten einer Zusammenarbeit werden in der Literatur behandelt. ${ }^{13}$

\section{Marktrealisierungen}

Die Marktrealisierungen hängen von der mittel- und langfristigen Zielstellung der deutschen Unternehmen ab. In Vietnam und allgemein in Asien sollen Unternehmen viel Geduld aufbringen und auch momentane Rückschläge verkraften können, um hohe wirtschaftliche Erfolge zu erzielen. Ausgehend von einer zumindest mittelfristigen Zielstellung sind folgende Grundziele möglich:

- Verkauf von Produkten in Vietnam

- Verkauf von Produkten in Südostasien

- Lohnfertigung

- Markterschließung in Vietnam, Produktion in Vietnam

- Markterschließung in Südostasien, Produktion in Vietnam

Ohne die genannten Ziele im einzelnen auszuführen, soll ein beispielhaftes Vorgehen in zwei Phasen skizziert werden:

1. Um den Markt und seine Möglichkeiten einschätzen zu können, beginnt das Unternehmen mit einer Repräsentation oder in einem Firmenpool. ${ }^{14}$ Erste Aktivitäten könnten die Betreuung der wichtigen Auslandsmessen in Hanoi und HCM-Stadt sowie die Organisation und Durchführung von Verkaufsausstellungen oder Präsentationen im Lande sein. Kontakte mit den entsprechenden Partnern werden hergestellt. Es ist sehr wichtig, die Produkte vorzustellen und Unterlagen in vietnamesischer Sprache bereitzuhalten.

2. Nach einer begründeten Einschätzung des Marktes besteht die zweite Phase in der Wahl einer Unternehmensform entsprechend den gesetzlichen Rahmenbedingungen. Die Kooperation wäre z. B. eine Möglichkeit, eine kostengünstige und schnell umsetzbare Zusammenarbeit mit einem vietnamesischen Partner zu realisieren. Die Kooperation wird aber meist mit geringen Einflußmöglichkeiten auf die Geschäftstätigkeit in Vietnam bezahlt. Beim JointVenture-Unternehmen wird das Kapital in Form von Geld, Krediten oder Sachmitteln, d. h. meist in Anlagen (die auch gebraucht sein können) zur Verfügung gestellt. Die Einflußnahme ist hier wesentlich größer. Die anderen Möglichkeiten werden hier nicht betrachtet. Grundsätzlich ist zu berücksichtigen, daß in allen Formen die gesetzlichen Bedingungen der Geschäftsführung für den ausländischen Partner Hindernisse und Hürden beinhalten.

\section{Zusammenfassung}

Dieser Beitrag gibt eine erste kurze Übersicht für Unternehmen, die geschäftlich mit Vietnam und/oder Südostasien tätig werden wollen. Probleme der Finanzierungen von Projekten, die bei einer detaillierten Betrachtung eine wesentliche Rolle spielen, wurden bewußt ausgeklammert. Dies gilt besonders für die hier im Vordergrund stehenden KMU. Durch die Finanzkrise in Südostasien ergeben sich weitere Gesichtspunkte, die aber die grundsätzliche Einschätzung nur bedingt beeinflussen. Ein Gesichtspunkt ist schon jetzt zu berücksichtigen. Die vietnamesische Währung - der Dong - hat ein festes Paritätsverhältnis von ca. 1 : 11.500 zum US-Dollar. Hier ist sicher mit einer Abwertung im Jahre 1998 zu rechnen, um die Überbewertung zum Dollar auszugleichen.

Ein weiterer wesentlicher Gesichtspunkt ist die Informationsbeschaffung. Die Daten von offiziellen vietnamesischen Institutionen sind statistisch nicht gut abgesichert. Die statistischen Erfassungen in Vietnam stehen erst am Anfang, so daß Daten nur als Relativ- bzw. Trendgrößen nutzbar sind. Hier ist die Situation mit der chinesischen vergleichbar.

Für ein Engagement in Vietnam sind gute einheimische Partner und viel Geduld nötig. Dann aber kann man mit hohen geschäftlichen Erfolgen in Vietnam und in Südostasien rechnen.

\section{Fußnoten}

1 Länderbericht-Vietnam, Berliner Bankgesellschaft, Okt. 1997

2 Südostasien aktuell, 5/1997, S. 437

3 Südostasien aktuell, 5/1997 S. 437

4 Märkte Ostasiens, 1995, Dt. Sparkassenverband S. 248

5 Vientiane Times, 4.-6.6.1997

6 Die Sektorgewichte aus Abb. 4 wurden zu Grunde gelegt und damit die Einzelrate berechnet.

7 Märkte Ostasiens, 1995, Dt. Sparkassenverband S. 245

8 Länderbericht-Vietnam, Okt. 1997, Berliner Bankgesellschaft

9 bfai-Info Asien, 15/1997

10 Kuihn, S., TFH Wildau, Diplomarbeit 1997

11 Berliner Zeitung, 3./4.1.1997

12 Law on Foreign Investment in Vietnam $(12.11 .1996)$ in Südostasien aktuell, 1/1997, S. 73

13 BMfW, Banken

$14 \mathrm{Zu}$ einem Firmenpool in Vietnam nähere Einzelheiten in dem von der VDWF Berlin organisierten Pool in Hanoi VDWF - Firmenpool, 10117 Berlin, Marienstr. 19-20

\section{Verfasser}

\section{Prof. Dr. Peter Gadow}

Technische Fachhochschule Wildau Institut für Internationales Marketing und Unternehmensfuihrung

Bahnhofstraße

15745 Wildau

Tel. +49 (0) 3375 - 508917 